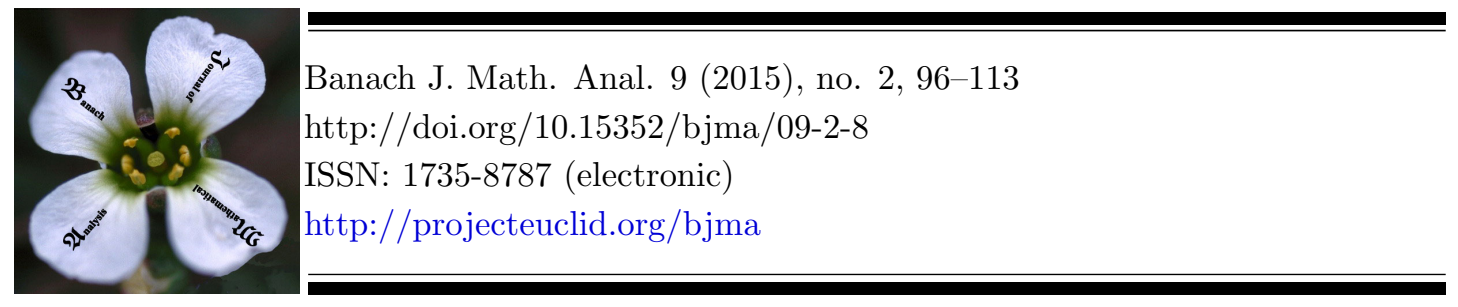

\title{
INTEGRATION THEORY FOR VECTOR VALUED FUNCTIONS AND THE RADON-NIKODYM THEOREM IN THE NON-ARCHIMEDEAN CONTEXT
}

\author{
JOSÉ N. AGUAYO* AND CAMILO G. PÉREZ
}

Communicated by R. E. Curto

\begin{abstract}
In this paper we define non-archimedean measures and integral operators taking values in a locally convex space. We show the relation between these two concept. We define what we called integral function respect to an integral operator. We give necessary and sufficient condition in order to know when a function is integrable with respect to an integral operator. In the second part, we define a kind of absolutely continuous relation between measures in this context. After that, we formulate a type of Radon-Nikodym Theorem between vector measures and a scalar measures which are absolutely continuous.
\end{abstract}

\section{Introduction and Notation}

In the second half of the 20th century, A. F. Monna and T. A. Springer [4] built the foundations of the Non-Archimedean Integration Theory. Both authors study certain functions defined on locally compact zero-dimensional topological spaces taking values on a non-archimedean valued field. Afterwards, in 1969, W. H. Schikhof and A. C. M. van Rooij devote their efforts to extend the theory to a larger class of topological spaces, so called zero-dimensional topological spaces. In 2001, J. N. Aguayo and a T. E. Gilsdorf, generalize this theory, considering vector measures and integrals with values in normed spaces [1].On the other hand, in 1971, Schikhof shows an analogue of the Radon-Nikodym's theorem in

Date: Received: Feb 3, 2014; Accepted: Jun. 22, 2014.

* Corresponding author.

2010 Mathematics Subject Classification. Primary 28B05; Secondary 28C15, 47G10.

Key words and phrases. scalar and vector measures, integral operators, absolutely continuous measures. 
the non-archimedean context. [6]. Later on, in 2001, Aguayo and Moraga extend the Schikhof work in the context of Banach spaces. [5].

The main goal of this paper, is to expand this theory for measures whose values are taken in locally convex spaces. Also, a Radon-Nikodym theorem is treated in this context.

Throughout this paper $\mathbb{K}$ will be a field with a non-trivial non-archimedean valuation $|\cdot|$ for which the metric space $\mathbb{K}$ is complete under the metric derived from this valuation, $E$ is a locally convex space over $\mathbb{K}$ whose topology is generated by a family of continuous seminorms denoted by $\Gamma$.

\section{Vector Measures and Integral operators}

Let $X$ be a non-empty set and $\Omega$ be a ring that cover $X$. As we know, $\Omega$ is a base for a topology $\tau$ on $X$ such that every element of $\Omega$ is clopen (closed and open) in $\tau$; hence $\tau$ is a zero-dimensional topology.

Definition 2.1. A finitely additive set function $m: \Omega \rightarrow E$ is said to be a vector measure on $\Omega$, or simply a measure, if:

[B] $\{m(U): U \in \Omega\}$ is bounded on $E$.

[M] for any net $\left(U_{\alpha}\right)_{\alpha \in I}$ of subsets in $\Omega$ such that $U_{\alpha} \downarrow \emptyset$ and any $V_{\alpha} \in \Omega$ such that $V_{\alpha} \subset U_{\alpha}$, then

$$
\lim _{\alpha} m\left(V_{\alpha}\right)=0
$$

Let $m$ be a vector measure on a ring $\Omega$ and $p \in \Gamma$. For an open subset $W$ of $X$ and for $x \in X$, we define

$$
\begin{aligned}
& \|W\|_{m, p}=\sup \{p(m(U)): U \in \Omega ; U \subset W\} \\
& \mathcal{N}_{m, p}(x)=\inf \left\{\|W\|_{m, p}: x \in W, W \in \tau\right\} .
\end{aligned}
$$

Most of the proofs of the properties for vector measures are similar to those proofs given by van Rooij and Schikhof [8], therefore those proofs will be omitted unless those proofs require a special treatment because the locally convex condition.

Lemma 2.2. If $m: \Omega \rightarrow E$ is a measure, $A$ and $B$ are open subsets in $X, x \in X$ and $p \in \Gamma$, then

(1) If $A \subset B$, then $\|A\|_{m, p} \leq\|B\|_{m, p}$.

(2) $\|A\|_{m, p}=\sup \left\{\|U\|_{m, p}: U \in \Omega, U \subset A\right\}$.

(3) $\|A \cup B\|_{m, p} \leq \max \left\{\|A\|_{m, p},\|B\|_{m, p}\right\}$.

(4) $\mathcal{N}_{m, p}(x)=\inf \left\{\|U\|_{m, p}: U \in \Omega ; x \in U\right\}$.

(5) $\mathcal{N}_{m, p}: X \rightarrow \mathbb{R}$ is upper semicontinuous.

Theorem 2.3. Let $m: \Omega \rightarrow E$ a finitely additive set function such that $\{m(V)$ : $V \in \Omega\}$ is a bounded set of $E$. Then, $m$ is a measure if, and only if, for each $p \in \Gamma$ and for any net $\left(U_{\alpha}\right)_{\alpha \in I}$ in $\Omega$ with $U_{\alpha} \downarrow \emptyset$,

$$
\lim _{\alpha}\left\|U_{\alpha}\right\|_{m, p}=0
$$


Proof. $(\Longleftarrow)$ It is obvious since

$$
p(m(V)) \leq\|U\|_{m, p} ; \text { for any } V \subset U, V \in \Omega .
$$

$(\Longrightarrow)$ Suppose that $m$ is a measure. Let $p \in \Gamma$ and $\left(U_{\alpha}\right)_{\alpha \in I}$ a net in $\Omega$ such that $U_{\alpha} \downarrow \emptyset$. We claim

$$
\lim _{\alpha}\left\|U_{\alpha}\right\|_{m, p}=0 .
$$

In fact, if $\left\|U_{\alpha_{0}}\right\|_{m, p}=0$ for some $\alpha_{0} \in I$, there is nothing to prove. Suppose $\left\|U_{\alpha}\right\|_{m, p}>0$ for all $\alpha \in I$ and choose $V_{\alpha} \subset U_{\alpha}, V_{\alpha} \in \Omega$, such that

$$
\frac{1}{2}\left\|U_{\alpha}\right\|_{m, p} \leq p\left(m\left(V_{\alpha}\right)\right) .
$$

Since $\lim _{\alpha} p\left(m\left(V_{\alpha}\right)\right)=0$, it follows that

$$
\lim _{\alpha}\left\|U_{\alpha}\right\|_{m, p}=0
$$

Theorem 2.4. For all open subset $W$ of $X$

$$
\|W\|_{m, p}=\sup \left\{\mathcal{N}_{m, p}(x): x \in W\right\} .
$$

Theorem 2.5. If $U \in \Omega$ and if $\delta>0$, then

$$
U_{\delta, p}:=\left\{x \in U: \mathcal{N}_{m, p}(x) \geq \delta\right\}
$$

is compact.

Theorem 2.6. Let $m_{1}, m_{2}: \Omega \rightarrow E$ two vector measures. Then, $m_{1}+m_{2}$ and $m_{1}-m_{2}$ are vector measures on $\Omega$. Furthermore, for all $p \in \Gamma$, we have

$$
\begin{gathered}
\mathcal{N}_{m_{1}+m_{2}, p} \leq \max \left\{\mathcal{N}_{m_{1}, p}, \mathcal{N}_{m_{2}, p}\right\} \\
\left|\mathcal{N}_{m_{1}, p}-\mathcal{N}_{m_{2}, p}\right| \leq \mathcal{N}_{m_{1}-m_{2}, p}
\end{gathered}
$$

Now, for a linear space $\mathcal{F}$ of $\mathbb{K}$-valued functions, the collection

$$
\Omega(\mathcal{F})=\left\{U \subset X: f \mathcal{X}_{U} \in \mathcal{F} \text { for all } f \in \mathcal{F}\right\}
$$

is a ring of sets that covers $X$ and $X \in \Omega$. As before, $\Omega$ is a base of zerodimensional topology $\tau(\mathcal{F})$. We call $\mathcal{F}$ a Wolfheze Space if every $f \in \mathcal{F}$ is $\tau(\mathcal{F})$-continuous and if for every $a \in X$ there exists $f \in \mathcal{F}$ with $f(a) \neq 0$. Under this conditions, $\tau(\mathcal{F})$ is the weakest topology for which every $f \in \mathcal{F}$ is continuous.

From now on, $\mathcal{F}$ will denote a Wolfheze space of $\mathbb{K}$-valued functions.

Definition 2.7. A lineal operator $I: \mathcal{F} \rightarrow E$ is said to be an Vector Integral Operator on $\mathcal{F}$, or simply, integral, if

[I] For any net $\left(f_{\alpha}\right)_{\alpha \in \Lambda}$ on $\mathcal{F}$ with $f_{\alpha} \downarrow 0$ and for each $g_{\alpha} \in \mathcal{F}$ such that $\left|g_{\alpha}\right| \leq\left|f_{\alpha}\right|$, it must have

$$
\lim _{\alpha} I\left(g_{\alpha}\right)=\theta
$$

in $E$, that is, for every $p \in \Gamma$,

$$
\lim _{\alpha} p\left(I\left(g_{\alpha}\right)\right)=0
$$


An equivalent formulation of $[I]$ is:

[I'] Let $\left(f_{\alpha}\right)_{\alpha \in \Lambda}$ be a net in $\mathcal{F}$ such that $f_{\alpha} \downarrow 0$. If $p \in \Gamma$ and if $\delta>0$, then there exists $\alpha=\alpha(p, \delta) \in \Lambda$ such that $p(I(g))<\delta$ for all $g \in \mathcal{F},|g| \leq\left|f_{\alpha}\right|$.

Let $I$ be an integral operator on $\mathcal{F}$. For $f \in \mathcal{F}$ we defined

$$
m_{f}: \Omega(\mathcal{F}) \rightarrow E, \quad m_{f}(U)=I\left(f \mathcal{X}_{U}\right) .
$$

This set function $m_{f}$ is well-defined, is finitely additive and is a vector measure. For the last statement, if $\left(U_{\alpha}\right)_{\alpha \in \Lambda}$ is an arbitrary net on $\Omega(\mathcal{F})$ with $U_{\alpha} \downarrow \emptyset$, then $f_{\alpha}=f \mathcal{X}_{U_{\alpha}} \downarrow 0$. We choose $V_{\alpha} \subset U_{\alpha}, V_{\alpha} \in \Omega(\mathcal{F})$ and put $g_{\alpha}=f \mathcal{X}_{V_{\alpha}}$. Clearly, $g_{\alpha} \in \mathcal{F}$ and $\left|g_{\alpha}\right| \leq\left|f_{\alpha}\right|$. Since $I$ is an operator, then

$$
\lim _{\alpha} m_{f}\left(V_{\alpha}\right)=\lim _{\alpha} I\left(g_{\alpha}\right)=\theta .
$$

On the other hand, if we suppose that

$$
\sup \left\{p\left(m_{f}(U)\right): U \in \Omega(\mathcal{F})\right\}=+\infty
$$

for some $p \in \Gamma$, then for a given $\pi \in \mathbb{K}$ with $|\pi|>1$, there exists a sequence $U(1), U(2), \cdots$ in $\Omega(\mathcal{F})$ such that $p\left(m_{f}(U(i))\right)=p\left(I\left(f \mathcal{X}_{U(i)}\right)\right) \geq|\pi|^{i}$ for all $i \in \mathbb{N}$. But $\pi^{-i} f \downarrow 0$ and $\left|\pi^{-i} f \mathcal{X}_{U(i)}\right| \leq\left|\pi^{-i} f\right|$, which implies $\lim I\left(\pi^{-i} f \mathcal{X}_{U(i)}\right)=0$. Thus, we have a contradiction.

Consequently, $m_{f}$ is a vector measure on $\Omega(\mathcal{F})$ since it satisfies $[M]$ and $[B]$.

In order to simplify the notation, we will write $\mathcal{N}_{f, p}$ instead of $\mathcal{N}_{m_{f}, p}$.

Lemma 2.8. If $p \in \Gamma, f, g \in \mathcal{F}$ and $a \in X$, then

$$
|f(a)| \mathcal{N}_{g, p}(a)=|g(a)| \mathcal{N}_{f, p}(a) .
$$

Proof. The collection $\Lambda=\{U \in \Omega(\mathcal{F}): a \in U\}$ is a directed set with the backward order inclusion. If $h=f(a) g-g(a) f$, then $h \mathcal{X}_{U} \downarrow 0$; hence, $\lim I\left(h \mathcal{X}_{U}\right)=0$, in other words, for a given $\delta>0$, there exists $U_{0} \in \Lambda$ such that $p\left(I\left(g \mathcal{X}_{V}\right)\right)<\delta$ for all $V \in \Omega, V \subset U_{0}$. By $\tau(\mathcal{F})$-upper semicontinuity of $\mathcal{N}_{f, p}$ we may assume that

$$
\forall x \in U_{0}: \mathcal{N}_{f, p}(x)<\mathcal{N}_{f, p}(a)+\delta .
$$

So, for $V \in \Omega, V \subset U_{0}$, we have

$$
p\left(f(a) m_{g}(V)-g(a) m_{f}(V)\right)=p\left(I\left(h \mathcal{X}_{V}\right)\right)<\delta
$$

and

$$
p\left(m_{f}(V)\right) \leq\|V\|_{m_{f}, p}=\sup \left\{\mathcal{N}_{f, p}(x): x \in V\right\} \leq \mathcal{N}_{f, p}(a)+\delta .
$$

Thus,

$$
\begin{aligned}
p\left(f(a) m_{g}(V)\right) & \leq \max \left\{p\left(f(a) m_{g}(V)-g(a) m_{f}(V)\right), p\left(g(a) m_{f}(V)\right)\right\} \\
& \leq \max \left\{\delta,|g(a)|\left[\mathcal{N}_{f, p}(a)+\delta\right]\right\}
\end{aligned}
$$

and, in consequence,

$$
|f(a)| \mathcal{N}_{g, p}(a) \leq|f(a)||| U_{0} \|_{m_{g}, p} \leq \max \left\{\delta,|g(a)|\left[\mathcal{N}_{f, p}(a)+\delta\right]\right\} .
$$

As $\delta$ was arbitrary, we obtain

$$
|f(a)| \mathcal{N}_{g, p}(a) \leq|g(a)| \mathcal{N}_{f, p}(a)
$$


By interchanging the roles of $f$ and $g$ and repeating the same argument, the lemma follows

Theorem 2.9. For every $p \in \Gamma$, there exists a unique function $\mathcal{N}_{I, p}: X \rightarrow[0, \infty[$ such that for all $f \in \mathcal{F}$

$$
|f| \mathcal{N}_{I, p}=\mathcal{N}_{f, p}
$$

Furthermore, $\mathcal{N}_{I, p}$ is $\tau(\mathcal{F})$-upper semicontinuous.

Corollary 2.10. For $f \in \mathcal{F}$, we define

$$
\|f\|_{I, p}=\|X\|_{m_{f}, p} .
$$

Then,

$$
\|f\|_{I, p}=\sup _{x \in X}|f(x)| \mathcal{N}_{I, p}(x)=\sup \{p(I(g)): g \in \mathcal{F},|g| \leq|f|\} .
$$

Moreover, for any $\delta>0,\left\{x \in X:|f(x)| \mathcal{N}_{I, p}(x) \geq \delta\right\}$ is $\tau(\mathcal{F})$-compact.

Proof. The first equality is directed from

$$
\|X\|_{m_{f}, p}=\sup _{x \in X} \mathcal{N}_{f, p}(x)
$$

and the previous theorem.

On the other hand, since $p(I(g)) \leq\|g\|_{I, p}$, we have

$$
\begin{aligned}
\|f\|_{I, p} & =\sup \{p(m(U)): U \in \Omega\}=\sup \left\{p\left(I\left(f \mathcal{X}_{U}\right)\right): U \in \Omega\right\} \\
& \leq \sup \{p(I(g)): g \in \mathcal{F},|g| \leq|f|\} \leq \sup \left\{\|g\|_{I, p}: g \in \mathcal{F},|g| \leq|f|\right\} \\
& \leq\|f\|_{I, p} .
\end{aligned}
$$

which establishes the formula. The last assertion follows from the facts: $X \in$ $\Omega(\mathcal{F}),|f| \mathcal{N}_{I, p}=\mathcal{N}_{f, p}$ and Theorem 2.5.

Corollary 2.11. For all $a \in X$, there exists $U \in \Omega(\mathcal{F})$ such that $a \in U$ and $\left\{x \in U: \mathcal{N}_{I, p}(x) \geq \delta\right\}$ is $\tau(\mathcal{F})$-compact for all $\delta>0$.

Summarizing, we have prove that if $I$ is an integral operator, then $\mathcal{N}_{I, p}$ is $\tau(\mathcal{F})$ upper semicontinuous and for all $f \in \mathcal{F}, \delta>0$, the set $\left\{x \in X:|f(x)| \mathcal{N}_{I, p}(x) \geq\right.$ $\delta\}$ is $\tau(\mathcal{F})$-compact. By $\Phi(\mathcal{F})$ we denote the collection of all functions $\phi: X \rightarrow$ $[0, \infty[$ which are $\tau(\mathcal{F})$-upper semicontinuous and satisfy

$$
(\forall f \in \mathcal{F})(\forall \delta>0)(\{x \in X:|f(x)| \phi(x) \geq \delta\} \text { is } \tau(F) \text {-compact }) .
$$

$\Phi(\mathcal{F}) \neq \emptyset$, since $\mathcal{N}_{I, p} \in \Phi(\mathcal{F})$ for all $p \in \Gamma$. Also, we can prove that $\Phi(\mathcal{F})$ is closed under supremum and infimum of finite collection of elements of $\Phi(\mathcal{F})$. Now, for $\phi \in \Phi(\mathcal{F})$ we denote

$$
\|f\|_{\phi}=\sup _{x \in X}|f(x)| \phi(x) .
$$

It is a routine to see that $\|\cdot\|_{\phi}$ is a non-archimedean seminorm on $\mathcal{F}$ and the collection $\left\{\|\cdot\|_{\phi}: \phi \in \Phi(\mathcal{F})\right\}$ generates a locally convex topology on $\mathcal{F}$ known as strict topology.

Theorem 2.12. Let $I: \mathcal{F} \rightarrow E$ a linear operator. The following conditions are equivalent: 
1. I is an integral operator.

2. I is strictly continuous.

3. Let $f \in \mathcal{F}$ and let $\left(f_{\alpha}\right)$ be a net in $\mathcal{F}$ such that $\left|f_{\alpha}\right| \leq|f|$ and $f_{\alpha} \rightarrow 0$ uniformly on $\tau$-compacts. Then, $\lim _{\alpha} I\left(f_{\alpha}\right)=0$.

Proof.

1.) $\Rightarrow$ 2.) Follows from the fact that for any $p \in \Gamma, \mathcal{N}_{I, p} \in \Phi(\mathcal{F})$ and for a given $f \in \mathcal{F}$,

$$
p(I(f))=p\left(I\left(f \mathcal{X}_{X}\right)\right)=p\left(m_{f}(X)\right) \leq\|X\|_{m_{f}, p}=\|f\|_{\mathcal{N}_{I, p}} .
$$

2.) \Rightarrow 3$.) Let $p \in \Gamma$ and $\varepsilon>0$. If $I$ is strictly continuous, there exists $\phi \in \Phi(\mathcal{F})$ and $\delta>0$ such that

$$
\|f\|_{\phi} \leq \delta \Longrightarrow p(I(f)) \leq \varepsilon .
$$

Take $f \in \mathcal{F}$ and $\left(f_{\alpha}\right)_{\alpha \in \Lambda}$ as in the assumptions. Set $Q=\{x \in X$ : $|f(x)| \phi(x) \geq \delta\}$. Since $\|\phi\|_{Q}=\sup _{x \in Q} \phi(x)<\infty$, there exists $\alpha_{0}$ such that

$$
\alpha \geq \alpha_{0} \Longrightarrow\left|f_{\alpha}(x)\right| \leq \frac{\delta}{\|\phi\|_{P}} \text { uniformly on } Q
$$

or equivalently,

$$
\alpha \geq \alpha_{0} \Longrightarrow\left|f_{\alpha}(x)\right| \phi(x) \leq \delta \text { for all } x \in Q .
$$

On the other hand, if $x \notin Q$, then

$$
\left|f_{\alpha}(x)\right| \phi(x) \leq|f(x)| \phi(x)<\delta .
$$

Therefore, for $x \in X$ and $\alpha \geq \alpha_{0}$ we have

$$
\left\|f_{\alpha}\right\|_{\phi}=\sup _{x \in X}\left|f_{\alpha}(x)\right| \phi(x) \leq \delta
$$

which implies $p\left(I\left(f_{\alpha}\right)\right) \leq \varepsilon$.

$3) \Rightarrow 1$ ) Follows from the Dini's Theorem.

Example 2.13. Let $X$ be a non-empty set and $\Omega$ a ring of subset of $X$ with $X \in \Omega$. Let $\mathcal{G}$ be the linear space generated by $\left\{\mathcal{X}_{U}: U \in \Omega\right\}$. Note that $\Omega=\Omega(\mathcal{G})$ and $\mathcal{G}$ is a Wolfheze space.

Let $m: \Omega \rightarrow E$ a finitely additive set function. Then, there exists a unique linear operator $I$ defined on $\mathcal{G}$ such that

$$
I\left(\mathcal{X}_{U}\right)=m(U) \text { for any } U \in \Omega .
$$

Moreover, $I$ is an integral operator if, and only if, $m$ is a measure. In fact, if $I$ is an integral, then we already know that for any $f \in \mathcal{G}, m_{f}$ is a measure, in particular, for $f \equiv \mathcal{X}_{X} \in \mathcal{G}$. Clearly, $m_{f}=m$.

On the other hand, if $m$ is a measure and $p \in \Gamma$, then $\mathcal{N}_{m, p} \in \Phi(\mathcal{G})$. For every $U \in \Omega$, we have

$$
p\left(I\left(\mathcal{X}_{U}\right)\right)=p(m(U)) \leq\|U\|_{m, p}=\left\|\mathcal{X}_{U}\right\|_{m, p}=\sup _{x \in U} \mathcal{N}_{m, p}(x) .
$$


If $g \in \mathcal{G}$, then $g=\sum_{i=1}^{n} \alpha_{i} \mathcal{X}_{U_{i}}$, for some $\alpha_{1}, \cdots \alpha_{n} \in \mathbb{K}$ and some $U_{1}, \cdots, U_{n} \in \Omega$, with $U_{i} \cap U_{j}=\emptyset, i \neq j$. Thus,

$$
\begin{aligned}
p(I(g)) & =p\left(I\left(\sum_{i=1}^{n} \alpha_{i} \mathcal{X}_{U_{i}}\right)\right)=p\left(\sum_{i=1}^{n} \alpha_{i} m\left(U_{i}\right)\right) \\
& \leq \max \left\{\left|\alpha_{i}\right| p\left(m\left(U_{i}\right)\right)\right\} \leq \max \left\{\sup _{x \in U_{i}}\left|\alpha_{i}\right| \mathcal{N}_{m, p}\right\} \\
& =\sup _{x \in X}|g(x)| \mathcal{N}_{m, p}(x)=\|f\|_{\mathcal{N}_{m, p} .}
\end{aligned}
$$

Therefore, $I$ is an integral operator.

Definition 2.14. Let $I: \mathcal{F} \rightarrow E$ be an integral operator. We say that $f: X \rightarrow \mathbb{K}$ is $I$-integrable if for every $\varepsilon>0$ and $p \in \Gamma$, there exists $h=h_{\varepsilon, p} \in \mathcal{F}$ such that

$$
\|f-h\|_{I, p} \leq \varepsilon
$$

We denote by $\mathcal{L}(I)$ the family of all $I$-integrable functions.

The next lemma may call an extension lemma.

Lemma 2.15. Let $Y$ be a non-empty subset of $X$ and let $Q \subset Y$ a $\tau$-compact. Then, for every $\tau$-continuous $f: Y \rightarrow \mathbb{K}$ and $\delta>0$, there exists $g \in \mathcal{F}$ such that $\|g\|_{X} \leq\|f\|_{Q},|g| \leq|f|$ on $Y$ and $\|g-f\|_{Q}<\delta$.

Proof. See [8] for more details.

Theorem 2.16. Let $f: X \rightarrow \mathbb{K}$ be a function. Then, $f$ is I-integrable if, and only if, satisfies the following two conditions:

1) $f$ is $\tau(\mathcal{F})$-continuous on each $X_{t, p}, t>0, p \in \Gamma$.

2) For every $\delta>0, p \in \Gamma$, there exists a $\tau(\mathcal{F})$-compact $Q_{\delta, p}$, contained is some $X_{t, p}$, such that $|f| \mathcal{N}_{I, p} \leq \delta$ on $X \backslash Q$.

Proof. Let $p \in \Gamma$. Then, there exists $\left(g_{n}\right)_{n \in \mathbb{N}}$ in $\mathcal{F}$ such that

$$
\lim _{n \rightarrow \infty}\left\|f-g_{n}\right\|_{I, p}=0 .
$$

Since $\lim g_{n}=f$ uniformly on $X_{t, p}$ we get that $f$ is $\tau(\mathcal{F})$-continuous on $X_{t, p}$.

For a $\delta>0$, we choose $g \in \mathcal{F}$ such that $\|f-g\|_{I, p} \leq \delta$. As we know,

$$
Q^{\prime}=\left\{x \in X:|g(x)| \mathcal{N}_{I, p}(x) \geq \delta\right\}
$$

is $\tau(\mathcal{F})$-compact. Take $t>0$ such that $t\|g\|_{Q^{\prime}}<\delta$ and $Q=Q^{\prime} \cap X_{t, p} . Q \tau(\mathcal{F})$ compact. Then, if $x \notin Q^{\prime}$, then $|g(x)| \mathcal{N}_{I, p}(x)<\delta$; if $x \in Q^{\prime} \backslash Q$, then $x \notin X_{t, p}$, which implies $\mathcal{N}_{I, p}(x)<t$. Thus, if $x \in X \backslash Q$,

$$
|f(x)| \mathcal{N}_{I, p}(x) \leq \max \left\{|f(x)-g(x)| \mathcal{N}_{I, p}(x),|g(x)| \mathcal{N}_{I, p}(x)\right\} \leq \max \left\{\delta,\left.t|| g\right|_{Q^{\prime}}\right\}<\delta \text {. }
$$

Conversely, let $f$ be that satisfies 1) and 2). Let $\delta>0$ and $p \in \Gamma$. Our purpose is to find $g \in \mathcal{F}$ with $\|f-g\|_{I, p} \leq \delta$. Take $Q$ and $t>0$ as in 2). $f$ and $\mathcal{N}_{I}$ are bounded on $Q$. Take $M>0$ such that $M \geq\|f\|_{Q}, M \geq\left\|\mathcal{N}_{I, p}\right\|_{Q}$ and consider $s=\min \left\{t, \delta M^{-1}\right\}$. Note that $Q \subset X_{t, p} \subset X_{s, p}$ and $f: X_{s, p} \rightarrow \mathbb{K}$ is $\tau(\mathcal{F})$-continuous. By Lemma 2.15, there exists $g \in \mathcal{F}$ such that $\|g\| \leq\|f\|_{Q}$, $|g| \leq|f|$ in $X_{s, p}$ and $\|g-f\|_{Q} \leq s$. 
Now, if $x \in Q$, then

$$
|f(x)-g(x)| \mathcal{N}_{I, p}(x) \leq s M \leq \delta
$$

if $x \in X_{s, p} \backslash Q$, then

$$
|f(x)-g(x)| \mathcal{N}_{I, p}(x) \leq|f(x)| \mathcal{N}_{I, p}(x) \leq \delta
$$

and if $x \notin X_{s, p}$, then

$$
\begin{aligned}
|f(x)-g(x)| \mathcal{N}_{I, p}(x) & \leq \max \left\{|f(x)| \mathcal{N}_{I, p}(x),|g(x)| \mathcal{N}_{I, p}(x)\right\} \\
& \leq \max \left\{\delta,\|f\|_{Q} s\right\}=\delta
\end{aligned}
$$

Therefore,

$$
\|f-g\|_{I, p} \leq \delta
$$

Corollary 2.17. If $f: X \rightarrow \mathbb{K}$ is bounded and $\tau(\mathcal{F})$-continuous, then $f$ is $I$ integrable.

Proof. It is enough to consider $f \neq 0$. Clearly, $f$ satisfies condition ( 1 ) in previous theorem. Let $\delta>0$ and $p \in \Gamma$. By Theorem 2.10,

$$
Q=\left\{x \in X:|f(x)| \mathcal{N}_{I, p}(x) \geq \delta\right\}
$$

is $\tau(\mathcal{F})$-compact. Moreover, taking $s=\frac{\delta}{\|f\|}$, then

$$
Q \subset\left\{x \in X: \mathcal{N}_{I, p}(x) \geq \frac{\delta}{\|f\|}\right\}=X_{s, p} .
$$

Thus, $f$ is $I$-integrable.

It is clear that $\mathcal{L}(I)$ is a $\mathbb{K}$-linear space. The corresponding covering ring $\Omega(\mathcal{L}(I))$ of $X$ generates a zero-dimensional topology $\tau(\mathcal{L}(I))$ on $X$. If $\mathcal{L}(I)$ is endowed with the locally convex topology generated by $\left\{\|\cdot\|_{I, p}\right\}_{p \in \Gamma}$, then $\mathcal{F}$ is dense on $\mathcal{L}(I)$. In consequence, there is a unique linear extension $I^{*}$ of $I$ to $\mathcal{L}(I)$ such that

$$
p\left(I^{*}(f)\right) \leq\|f\|_{I, p} \text { for any } p \in \Gamma \text { and } f \in \mathcal{L}(I) .
$$

Let us denote by

$$
\Omega^{*}=\left\{V \subset X: V \cap X_{t, p} \text { is a } \tau(F) \text {-clopen on } X_{t, p} \text {, for all } t>0 \text { and } p \in \Gamma\right\}
$$

$\Omega^{*}$ is a covering ring of $X$ with $X \in \Omega^{*}$. We denote by $\tau^{*}$ the zero-dimensional topology generate by $\Omega^{*}$ on $X$. It follows from Theorem 2.16, that $\Omega^{*} \subset \Omega(\mathcal{L}(I))$. Obviously, $\tau^{*}$ is finer than $\tau(\mathcal{F})$ and $\tau(\mathcal{L}(I))$ is finer than $\tau^{*}$.

Lemma 2.18. $f: X \rightarrow \mathbb{K}$ is $\tau^{*}$-continuous if, and only if, it is $\tau(\mathcal{F})$-continuous on every $X_{t, p}$.

Proof. It follows from the fact that every $\tau^{*}$-open set is the union of a collection of elements of $\Omega^{*}$.

Theorem 2.19. $\mathcal{L}(I)$ is a Wolfheze space and $I^{*}$ is an integral operator on $\mathcal{L}(I)$. 
Proof. Let $f \in \mathcal{L}(I)$. By Theorem 2.16 and Lemma 2.18, $f$ is $\tau^{*}$-continuous and then $\tau(\mathcal{L}(I))$-continuous. From the fact that $\mathcal{F} \subset \mathcal{L}(I)$, it follows that $\mathcal{L}(I)$ is a Wolfheze space. It remains to prove that $I^{*}$ is an integral. Since $\Omega(\mathcal{F}) \subset \Omega(\mathcal{L}(I))$, $\mathcal{N}_{I, p}$ is $\tau(\mathcal{L}(I))$-upper semicontinuous. On the other hand, $\tau(\mathcal{L}(I))$ is the weakest topology that makes continuous every $f \in \mathcal{L}(I)$, which implies $\tau^{*}=\tau(\mathcal{L}(I))$. Since $\tau^{*}$ and $\tau(\mathcal{F})$ induce the same topology on $X_{t, p}$ we see that $\mathcal{N}_{I, p} \in \Phi(\mathcal{L}(I))$. Therefore, $I^{*}$ is strictly continuous, and the theorem follows.

Corollary 2.20. $\mathcal{N}_{I^{*}, p}=\mathcal{N}_{I, p}$ for every $p \in \Gamma, \mathcal{L}\left(I^{*}\right)=\mathcal{L}(I)$ and $I^{* *}=I^{*}$.

Proof. Let us denote by $\Psi$ the set of all functions $\psi: X \rightarrow \mathbb{R}^{+} \cup\{0\}$ such that

- $\psi$ is $\tau(\mathcal{L}(I))$-upper semicontinuous,

- $\forall p \in \Gamma, p\left(I^{*}(f)\right) \leq\|f\|_{\psi}, f \in \mathcal{L}(I)$.

$\Psi \neq \varnothing$, since $\mathcal{N}_{I, p} \in \Psi$. Let $\psi \in \Psi, a \in X$ and $\varepsilon>0$. Choose $U \in \Omega(\mathcal{L}(I))$ such that $a \in U$ and $\psi(x) \leq \psi(a)+\varepsilon$ for any $x \in U$. Take $f \in \mathcal{L}(I)$ with $|f(a)|=1$ and $|f| \leq 1$. Note that $\mathcal{X}_{U} f \in \mathcal{L}(I)$ and

$$
p\left(I^{*}\left(\mathcal{X}_{U} f\right)\right) \leq\left\|\mathcal{X}_{U} f\right\|_{\psi}
$$

By Corollary 2.10, we have

$$
\begin{aligned}
\left\|\mathcal{X}_{U} f\right\|_{I^{*}, p} & =\sup \left\{p\left(I^{*}(g)\right): g \in \mathcal{L}(I),|g| \leq\left|\mathcal{X}_{U} f\right|\right\} \\
& \leq \sup \left\{\|g\|_{\psi}: g \in \mathcal{L}(I),|g| \leq\left|\mathcal{X}_{U} f\right|\right\} \leq\left\|\mathcal{X}_{U} f\right\|_{\psi}
\end{aligned}
$$

which implies that

$$
\begin{aligned}
\mathcal{N}_{I^{*}, p}(a) & \leq \sup _{x \in U}|f(x)| \mathcal{N}_{I^{*}, p}(x) \leq \sup _{x \in U}|f(x)| \psi(x) \\
& \leq \sup _{x \in U} \psi(x) \leq \psi(a)+\varepsilon
\end{aligned}
$$

Since $\varepsilon$ was arbitrary, we conclude that $\mathcal{N}_{I^{*}, p}(a) \leq \psi(a)$. Since $\mathcal{N}_{I, p} \in \Psi$, we get $\mathcal{N}_{I^{*}, p} \leq \mathcal{N}_{I, p}$.

In order to prove the conversely inequality, suppose that there exists $a \in X$ and $t>0$ such that $\mathcal{N}_{I^{*}, p}(a)<t<\mathcal{N}_{I, p}(a)$. By Corollary 2.11 and by the fact that $\tau(\mathcal{L}(I))=\tau^{*}$, we can choose $U \in \Omega^{*}$ with $a \in U$ such that $U \subset\{x \in X$ : $\left.\mathcal{N}_{I^{*}, p}(x)<t\right\}$. Note that $\left\{x \in U: \mathcal{N}_{I^{*}, p}(x) \geq \delta\right\}$ is $\tau(\mathcal{L}(I))$-compact for any $\delta>0$. As $X_{t, p}$ is $\tau(\mathcal{F})$-closed on $X$ and $U \cap X_{t, p}$ is $\tau(\mathcal{F})$-clopen on $X_{t, p}$, then $U \cup\left(X \backslash X_{t, p}\right)$ is $\tau$-open. If $f \in \mathcal{F},|f(a)|=1$ and $|f| \leq 1$, we have

$$
\begin{aligned}
t & <|f(a)| \mathcal{N}_{I, p}(a) \\
& =\mathcal{N}_{m_{f}, p}(a) \leq\left\|U \cup\left(X \backslash X_{t, p}\right)\right\|_{m_{f}, p} \\
& =\sup \left\{p\left(m_{f}(W)\right): W \in \Omega, W \subset U \cup\left(X \backslash X_{t, p}\right)\right\}
\end{aligned}
$$

which implies that there exists $W \in \Omega, W \subset U \cup\left(X \backslash X_{t, p}\right)$ such that

$$
p\left(I\left(\mathcal{X}_{W} f\right)\right)=p\left(m_{f}(W)\right)>t .
$$


Since $\mathcal{X}_{W} f \in \mathcal{F} \subset \mathcal{L}(I)$ and $U \in \Omega^{*} \subset \Omega(\mathcal{L}(I))$, we have $\mathcal{X}_{U}\left(\mathcal{X}_{W} f\right)=\mathcal{X}_{U \cap W} f \in$ $\mathcal{L}(I)$. It follows that

$$
\begin{aligned}
p\left(I^{*}\left(\mathcal{X}_{W} f-\mathcal{X}_{U \cap W} f\right)\right) & \leq\left\|\mathcal{X}_{W} f-\mathcal{X}_{U \cap W} f\right\|_{I, p}=\sup _{x \in X}\left|\mathcal{X}_{W \backslash U} f(x)\right| \mathcal{N}_{I, p}(x) \\
& \leq \sup _{x \in X}\left|\mathcal{X}_{W \backslash U}\right| \mathcal{N}_{I, p}(x) ; \quad(|f| \leq 1) \\
& =\sup _{x \in W \backslash U} \mathcal{N}_{I, p}(x) \leq \sup _{x \in X \backslash X_{t, p}} \mathcal{N}_{I, p}(x) ; \quad\left(W \backslash U \subset X \backslash X_{t, p}\right) \\
& <t .
\end{aligned}
$$

Therefore,

$$
p\left(I^{*}\left(\mathcal{X}_{W} f-\mathcal{X}_{U \cap W} f\right)\right)<t<p\left(I\left(\mathcal{X}_{W} f\right)\right) .
$$

Now, $\mathcal{X}_{W} f \in \mathcal{F}$ implies that $I^{*}\left(\mathcal{X}_{W} f\right)=I\left(\mathcal{X}_{W} f\right)$ which means that

$$
p\left(I^{*}\left(\mathcal{X}_{W} f-\mathcal{X}_{U \cap W} f\right)\right)<t<p\left(I^{*}\left(\mathcal{X}_{W} f\right)\right),
$$

But,

$$
t<p\left(I^{*}\left(\mathcal{X}_{U \cap W} f\right)\right) \leq \sup _{x \in W \cap U}|f(x)| \mathcal{N}_{I^{*}, p}(x) \leq \sup _{x \in U}|f(x)| \mathcal{N}_{I^{*}, p}(x) \leq t
$$

which is impossible. We conclude that $\mathcal{N}_{I^{*}, p} \geq \mathcal{N}_{I, p}$.

\section{The Radon-Nikodym Theorem}

The purpose of this section is to give a version of the Radon-Nikodym Theorem for locally convex spaces in the non-archimedean context.

In order to do that, we will need to enlarge the range of our functions. Until previous sections, the range of our functions were the non-archimedean field $\mathbb{K}$. Now, the range of them will be a locally convex spaces $E$ over $\mathbb{K}$.

Let $\mathcal{F}(X, E)$ be a linear vector space of functions defined from a set $X$ into $E$. We define

$$
\Omega(\mathcal{F}(X, E))=\left\{U \subset X: \mathcal{X}_{U} \otimes f \in \mathcal{F}(X, E), \text { for any } f \in \mathcal{F}(X, E)\right\}
$$

As before, $\Omega(\mathcal{F}(X, E))$ is a ring such that $X \in \Omega(\mathcal{F}(X, E))$ and generates a zero-dimensional topology $\tau(\mathcal{F}(X, E))$ in $X$. If each of functions in $\mathcal{F}(X, E)$ are continuous in this new topology and for each $x \in X$ there exists $f \in \mathcal{F}(X, E)$ such that $f(x) \neq 0$, then we will say that $\mathcal{F}(X, E)$ is a Wolfheze space. This topology is the weakest topology that make every $f \in \mathcal{F}(X, E)$ continuous.

Now, suppose that $X$ is provided with a zero-dimensional topology $\tau$. We already know that the collection $\Omega$ of all $\tau$-clopen subsets of $X$ forms a ring, $\mathcal{F}(X)=\left\langle\left\{\mathcal{X}_{U}: U \in \Omega\right\}\right\rangle$ is a Wolfheze space and $\tau(\Omega(\mathcal{F}))=\tau$. Even more, if $\mu: \Omega \rightarrow \mathbb{K}$ is a scalar measure, then the linear operator $I: \mathcal{F}(X) \rightarrow K$ defined by $I\left(\mathcal{X}_{U}\right)=\mu(U)$ is an integral operator. By the fact that $X \in \Omega, \mathcal{N}_{I}=\mathcal{N}_{\mu}$.

On the other hand, if we consider

$$
\mathcal{F}(X, E)=\left\langle\left\{\mathcal{X}_{U} \otimes e: U \in \Omega, e \in E\right\}\right\rangle,
$$

where $\mathcal{X}_{U} \otimes e(x)=\mathcal{X}_{U}(x) \otimes e$, then $\Omega(\mathcal{F}(X, E))=\tau(\Omega(\mathcal{F}))=\tau$ and $\mathcal{F}(X, E)$ is a Wolfheze space. 
For a fixed $p \in \Gamma$, we put

$$
\|g\|_{\mu, p}=\sup _{x \in X} p(g(x)) \mathcal{N}_{I}(x)
$$

for a function $g: X \rightarrow E$. Of course, for any $g \in \mathcal{F}(X, E),\|g\|_{\mu, p}<\infty$.

Definition 3.1. Let $g: X \rightarrow \mathrm{E}$ a function. We will say that $g$ is $\mu$-integrable if for any $p \in \Gamma$ and $\varepsilon>0$, there exists $h=h(\varepsilon, p) \in \mathcal{F}(X, E)$ such that

$$
\|g-h\|_{\mu, p}<\varepsilon
$$

The integrable functions form a linear space $\mathcal{L}(\mu, E)$ that contains $\mathcal{F}(X, E)$. Note that if $g \in \mathcal{L}(\mu, E)$, then $\|g\|_{\mu, p}<\infty$ and $\|\cdot\|_{\mu, p}$ is a semi-norm on $\mathcal{L}(\mu, E)$,

for all $p \in \Gamma$. The family of all seminorms $\left\{\|\cdot\|_{\mu, p}\right\}_{p \in \Gamma}$ generates a locally convex topology on $\mathcal{L}(\mu, E)$.

In order to give another characterization of $\mu$-integrable functions, we need the following lemma whose arguments to prove it, are similar to those arguments given in Lemma 3.1 [8].

Lemma 3.2. Let $Y \subset X$ and $Q$ be a $\tau(\mathcal{F}(X, E))$-compact subset of $Y$. For any $\tau(\mathcal{F}(X, E))$-continuous function $f: Y \rightarrow E$, any $p \in \Gamma$ and every $\delta>0$ there exists $g \in \mathcal{F}(X, E)$ such that

- $\|g\|_{X, p}=\sup _{x \in X} p(g(x)) \leq\|f\|_{Q, p}=\sup _{x \in Q} p(f(x))$,

- $p(g) \leq p(f)$ in $Y$, and

- $\|g-f\|_{Q, p} \leq \delta$.

Theorem 3.3. A function $f: X \rightarrow E$ is $\mu$-integrable if and only if it satisfies the following conditions:

(1) $f$ is $\tau(\mathcal{F}(X, E))$-continuous on each $X_{t}=\left\{x \in X: \mathcal{N}_{\mu}(x) \geq t\right\}, t>0$.

(2) For every $\delta>0$ and $p \in \Gamma$, there exists a $\tau(\mathcal{F}(X, E))$-compact $Q$, contained in some $X_{t}$, such that $p(f) \mathcal{N}_{\mu} \leq \delta$ off $Q$.

Proof. Let $f \in \mathcal{L}(\mu, E)$ and $p \in \Gamma$. There exists a sequence $\left(g_{n}\right)_{n \in \mathbb{N}}$ in $\mathcal{F}(X, E)$ such that $\lim _{n \rightarrow \infty}\left\|f-g_{n}\right\|_{\mu, p}=0$. Now, if $t>0$ and $x \in X_{t}$, then

$$
p\left(g_{n}(x)-f(x)\right)=\frac{p\left(g_{n}(x)-f(x)\right) \mathcal{N}_{\mu}(x)}{\mathcal{N}_{\mu}(x)} \leq \frac{1}{t}\left\|g_{n}-f\right\|_{\mu, p} .
$$

which means that $\left(g_{n}\right)_{n \in \mathbb{N}}$ converges uniformly to $f$ in $X_{t}$. From this, $f$ satisfies (1). Let $\delta>0$; hence there exists $g \in \mathcal{F}(X, E)$ such that $\|f-g\|_{\mu, p} \leq \delta$. The set

$$
R=\left\{x \in X: p(g(x)) \mathcal{N}_{\mu}(x) \geq \delta\right\}
$$

is compact in $X$.

If we choose $t>0$ such that $t\|g\|_{R, p}<\delta$, then $Q=R \cap X_{t}$ is also a compact in $X$. A simple calculation proves $p(f(x)) \mathcal{N}_{\mu}(x) \leq \delta$ for $x \in X \backslash Q$.

Conversely, assume (1) and (2). Take $\delta>0$; we shall construct a $g \in \mathcal{F}(X, E)$ such that $\|f-g\|_{\mu, p} \leq \delta$. Let $Q, t$ be as in (2). Both functions, $f$ and $\mathcal{N}_{\mu}$, are bounded on $Q$. Let $M>0, M \geq\|f\|_{Q, p}, M \geq\left\|\mathcal{N}_{\mu}\right\|_{Q, p}$. Let $s=\min \left\{t, \delta M^{-1}\right\}$. Note that $Q \subset X_{t} \subset X_{s}$ and $f: X_{s} \rightarrow E$ is continuous. By the preceding lemma, 
there exists $g \in \mathcal{F}(X, E)$ such that $\|g\|_{X, p} \leq\|f\|_{Q, p}, p(g) \leq p(f)$ in $X_{s}$ and $\|g-f\|_{Q, p} \leq s$. Thus, if $x \in Q$, then

$$
p(f(x)-g(x)) \mathcal{N}_{\mu}(x) \leq s M \leq \delta,
$$

if $x \in X_{s} \backslash Q$, then

$$
p(f(x)-g(x)) \mathcal{N}_{\mu}(x) \leq p(f(x)) \mathcal{N}_{\mu}(x) \leq \delta
$$

and finally, if $x \notin X_{s}$, then

$$
\begin{aligned}
p(f(x)-g(x)) \mathcal{N}_{\mu}(x) & \leq \max \left\{p(f(x)) \mathcal{N}_{\mu}(x), p(g(x)) \mathcal{N}_{\mu}(x)\right\} \\
& \leq \max \left\{\delta,\|f\|_{Q, p} s\right\}=\delta .
\end{aligned}
$$

Therefore,

$$
\|f-g\|_{\mu, p} \leq \delta
$$

Before giving definitions of certain technical limits, we need the following lemma that will help make sense of those limits. The proof was held in [6].

Lemma 3.4. Let $\mu: \Omega \rightarrow \mathbb{K}$ a scalar measure. Then, if $a \in X$ and $c \in] 0,1[$, then for every neighborhood $U$ of a there exists a neighborhood $W \in \Omega$ of a such that $W \subset U$ and $|\mu(W)| \geq \operatorname{cN}_{\mu}(a)$.

Definition 3.5. Let $\theta: \Omega \rightarrow E$ a set function and $\mu: \Omega \rightarrow \mathbb{K}$ a scalar measure. For $a \in X, e \in E, c \in] 0,1[$ and $r \in \mathbb{R}$ we write:

(i)

if

$$
\underset{U \rightarrow a}{\operatorname{LIM}} \theta(U)=e
$$

$(\forall p \in \Gamma)(\forall \varepsilon>0)(\exists U \in \Omega, a \in U)(V \subset U, V \in \Omega \Rightarrow p(\theta(V)-e) \leq \varepsilon) ;$

(ii)

if

$$
\underset{U \rightarrow a}{\operatorname{LIM}_{\mu, c}} \theta(U)=e
$$

$(\forall p \in \Gamma)(\forall \varepsilon>0)(\exists U \in \Omega, a \in U)\left(V \subset U, V \in \Omega,|\mu(V)| \geq c \mathcal{N}_{\mu}(a) \Rightarrow p(\theta(V)-e) \leq \varepsilon\right) ;$

(iii)

if

$$
\underset{U \rightarrow a}{\operatorname{LIM}_{\mu}} \theta(U)=e
$$

$$
\operatorname{LIM}_{U \rightarrow a} \theta(U)=e
$$

for all $c \in] 0,1[$;

(iv) For fixed $p \in \Gamma$,

$$
\overline{\operatorname{LIM}_{U \rightarrow a}} p(\theta(U))=r
$$

if

$$
(\forall \varepsilon>0)(\exists U \in \Omega, a \in U)(r-\varepsilon \leq \sup \{p(\theta(V)): V \in \Omega, V \subset U\} \leq r+\varepsilon)
$$

Lemma 3.6. Let $m: \Omega \rightarrow E$ a vectorial measure and $a \in X$. Then, 
(1) $\mathcal{N}_{m, p}(a)=\overline{L I M} p(\theta(U))$

(2) $\forall p \in \Gamma, \mathcal{N}_{m, p}(a)=0 \Longleftrightarrow L I M_{U \rightarrow a} m(U)=0$

(3) Let $\mu: \Omega \rightarrow \mathbb{K}$ a scalar measure and $0<c<1$. If $\underset{U \rightarrow a}{\operatorname{LI} M_{\mu, c}} m(U)=0$, then $\underset{U \rightarrow a}{\operatorname{LIMm}}(U)=0$

Proof. To prove (1.), it is enough to prove that

$$
(\forall \varepsilon>0)(\exists U \in \Omega, a \in U)\left(\mathcal{N}_{m, p}(a)-\varepsilon \leq\|U\|_{m, p} \leq \mathcal{N}_{m, p}(a)+\varepsilon\right)
$$

or equivalently

$$
(\forall \varepsilon>0)(\exists U \in \Omega, a \in U)\left(\left|\|U\|_{m, p}-\mathcal{N}_{m, p}(a)\right| \leq \varepsilon\right)
$$

which follows directed from the definition of $\mathcal{N}_{m, p}$.

To show (2.), we need to follow the definitions of $\mathcal{N}_{m, p}$ and $L I M_{U \rightarrow a} m(U)$, respectively.

To prove (3.), let $\varepsilon>0$. By definition, there exists $U \in \Omega$ such that if $a \in U$, $V \in \Omega, V \subset U$ and $|\mu(V)| \geq c \mathcal{N}_{\mu}(a)$, then $p(m(V)) \leq \varepsilon$.

Take $W \in \Omega$ such that $W \subset U$, but $|\mu(W)|<\mathcal{N}_{\mu}(a)$. By Lemma 3.4, we may assume that $|\mu(U)| \geq c \mathcal{N}_{\mu}(a)$. Then $|\mu(U \backslash W)|=|\mu(U)| \geq c \mathcal{N}_{\mu}(a)$, therefore

$$
p(m(W))=p(m(U)-m(U \backslash W)) \leq \max \{p(m(U)), p(m(U \backslash W))\} \leq \varepsilon
$$

Next, we define a vector measure that will be crucial in what follows. For a scalar measure $\mu$, we consider the linear operator $T_{\mu}: \mathcal{F}(X, E) \rightarrow E$ defined by

$$
T_{\mu}\left(\mathcal{X}_{U} \otimes e\right)=\mu(U) e .
$$

We claim that this operator satisfies

$$
\forall p \in \Gamma, \quad p\left(T_{\mu}(f)\right) \leq\|f\|_{\mu, p}, \quad(f \in \mathcal{F}(X, E)) .
$$

In fact, for $p \in \Gamma$ :

$$
\begin{aligned}
p\left(T_{\mu}(f)\right) & =p\left(T_{\mu}\left(\sum_{i=1}^{n} \mathcal{X}_{U_{i}} \otimes e_{i}\right)\right) \leq \max \left\{p\left(\mu\left(U_{i}\right) e_{i}\right): 1 \leq i \leq n\right\} \\
& =\max \left\{\left|\mu\left(U_{i}\right)\right| p\left(e_{i}\right): 1 \leq i \leq n\right\} \leq\left\{\left\|U_{i}\right\|_{\mu} p\left(e_{i}\right): 1 \leq i \leq n\right\} \\
& =\max \left\{\sup _{x \in U_{i}} p\left(e_{i}\right) \mathcal{N}_{\mu}(x): 1 \leq i \leq n\right\}=\sup _{x \in X} p(f(x)) \mathcal{N}_{\mu}(x)=\|f\|_{\mu, p}
\end{aligned}
$$

Now, since $\mathcal{F}(X, E)$ is dense in $\mathcal{L}(\mu, E)$ (with respect to the family $\left\{\|\cdot\|_{\mu, p}\right\}_{p \in \Gamma}$ ), the operator $T_{\mu}$ may be continuously extended to $\mathcal{L}(\mu, E)$, satisfying

$$
\forall p \in \Gamma, \quad p\left(T_{\mu}(f)\right) \leq\|f\|_{\mu, p}, \quad(f \in \mathcal{L}(\mu, E)) .
$$

On the other hand, if we fix $g \in \mathcal{L}(\mu, E)$, with $\|g\|_{X, p}=\sup p(g(x))<+\infty$ for all $p \in \Gamma$, then we define

$$
S_{g}: \mathcal{L}(\mu) \rightarrow E, \quad S_{g}(f)=T_{\mu}(f \otimes g)
$$


By Theorem 2.12, $S_{g}$ is an integral operator, since if $f \in \mathcal{L}(\mu)$ and $p \in \Gamma$, then

$$
\begin{aligned}
p\left(S_{g}(f)\right) & =p\left(T_{\mu}(f \otimes g)\right) \leq\|f \otimes g\|_{\mu, p}=\sup _{x \in X} p(f(x) g(x)) \mathcal{N}_{\mu}(x) \\
& \leq\|g\|_{X, p} \sup _{x \in X}|f(x)| \mathcal{N}_{\mu}(x)=\|g\|_{X, p}\|f\|_{\mu} .
\end{aligned}
$$

Thus, by the example ??, there exists a unique vector measure $m_{g}: \Omega \rightarrow E$ associated to $S_{g}$ such that

$$
m_{g}(U)=S_{g}\left(\mathcal{X}_{U}\right)=T_{\mu}\left(\mathcal{X}_{U} \otimes g\right)
$$

Let us denote $m_{g}$ by $\mu \otimes g$. Note that if $g=e$, then $\mu \otimes e(U)=\mu(U) e$, thus the new notation makes sense. At the same time, if $p \in \Gamma, U \in \Omega$ and $x \in X$, then

$$
\begin{aligned}
& \|U\|_{\mu \otimes g, p} \\
& \quad=\sup \{p(\mu \otimes g(V)): V \subset U, V \in \Omega\}=\sup \left\{p\left(S_{g}\left(\mathcal{X}_{V}\right)\right): V \subset U, V \in \Omega\right\} \\
& \quad \leq \sup \left\{\|g\|_{X}\left\|\mathcal{X}_{V}\right\|_{\mu}: V \subset U, V \in \Omega\right\}=\|g\|_{X, p} \sup \left\{\left\|\mathcal{X}_{V}\right\|_{\mu}: V \subset U, V \in \Omega\right\} \\
& \quad=\|g\|_{X, p} \sup \left\{\|V\|_{\mu}: V \subset U, V \in \Omega\right\}=\|g\|_{X, p}\|U\|_{\mu} .
\end{aligned}
$$

and

$$
\begin{aligned}
\mathcal{N}_{\mu \otimes g, p}(x) & =\inf \left\{\|W\|_{\mu \otimes g, p}: x \in W, W \in \Omega\right\} \leq\left\{\|g\|_{X}\|W\|_{\mu}: x \in W, W \in \Omega\right\} \\
& =\|g\|_{X, p} \inf \left\{\|W\|_{\mu}: x \in W, W \in \Omega\right\}=\|g\|_{X, p} \mathcal{N}_{\mu}(x) .
\end{aligned}
$$

Lemma 3.7. If $\mu$ is a scalar measure and $g \in \mathcal{L}(\mu, E)$, then

$$
\underset{U \rightarrow a}{\operatorname{LIM}}[\mu \otimes g(U)-\mu \otimes g(a)(U)]=0 .
$$

Proof. Let $p \in \Gamma, \varepsilon>0$ and $a \in X$. Note that

$$
\mu \otimes g(U)-\mu \otimes g(a)(U)=\mu \otimes[g-g(a)](U) .
$$

With out loss of generality, we may assume that $g(a)=0$ and $\mathcal{N}_{\mu}(a) \leq 1$. Since $g$ is $\mu$-integrable, by Theorem 3.3, there exists a compact $Q$ of $X$ contained in some $X_{t}$ such that $p(g(x)) \mathcal{N}_{\mu}(x) \leq \varepsilon$ for all $x \in X \backslash Q$. Now, if $a \notin Q$, then we choose $U \in \Omega$ such that $a \in U$ and $U \subset X \backslash Q$; or if $a \in Q$, then we choose $U \in \Omega$ such that

$$
x \in U \cap X_{t} \Longrightarrow p(g(x)) \leq \varepsilon .
$$

In both cases we have

$$
\forall x \in U, \quad p(g(x)) \mathcal{N}_{\mu}(x) \leq \varepsilon .
$$

Therefore, if $V \in \Omega, V \subset U$, then

$$
\begin{aligned}
p(\mu \otimes g(V)) & =p\left(T_{\mu}\left(\mathcal{X}_{V} \otimes g\right)\right) \leq\left\|\mathcal{X}_{V} \otimes g\right\|_{\mu, p} \\
& =\sup _{x \in X} p\left(\mathcal{X}_{V} \otimes g(x)\right) \mathcal{N}_{\mu}(x)=\sup _{x \in V} p(g(x)) \mathcal{N}_{\mu}(x) \leq \varepsilon
\end{aligned}
$$

Since $p \in \Gamma$ was arbitrary, we are done 
Definition 3.8. Let $m: \Omega \rightarrow E$ a vector measure and $\mu: \Omega \rightarrow \mathbb{K}$ a scalar measure. We will say that $m$ is absolutely continuous with respect to $\mu$ if, for any $a \in X$, there exists $e_{a} \in E$ such that

$$
\forall p \in \Gamma, \quad \mathcal{N}_{m-\mu \otimes e_{a}, p}(a)=0 .
$$

In that case we will write $m<<\mu$.

Remark 3.9. If $m<<\mu$, then for every $U \in \Omega$, we have

$$
\|U\|_{\mu}=0 \Longrightarrow \forall p \in \Gamma,\|U\|_{m, p}=0 \text {. }
$$

Proof. First of all, note that

$$
\mathcal{N}_{\mu}(a)=0 \Rightarrow \forall p \in \Gamma, \mathcal{N}_{m, p}(a)=0 .
$$

In fact, if $a \in X$ such that $\mathcal{N}_{\mu}(a)=0$ and $p \in \Gamma$, then there exists $e_{a} \in E$ such that $\mathcal{N}_{m-\mu \otimes e_{a}, p}(a)=0$. Also, by a previous results,

$$
\mathcal{N}_{\mu \otimes e_{a}, p}(a) \leq\left\|e_{a}\right\|_{X} \mathcal{N}_{\mu}(a)=p\left(e_{a}\right) \mathcal{N}_{\mu}(a)=0 .
$$

Thus, by Theorem 2.6,

$$
0 \leq \mathcal{N}_{m, p}(a)=\mathcal{N}_{m-\mu \otimes e_{a}+\mu \otimes e_{a}, p}(a) \leq \max \left\{\mathcal{N}_{m-\mu \otimes e_{a}, p}(a), \mathcal{N}_{\mu \otimes e_{a}, p}(a)\right\}=0 .
$$

Now, if $U \in \Omega$ such that $\|U\|_{\mu}=0$ and $x \in X$, then $\mathcal{N}_{\mu}(x)=0$; hence $\mathcal{N}_{m, p}(x)=$ 0 . Therefore, $\|U\|_{m, p}=0$.

Theorem 3.10. (Radon-Nikodym) Let $m: \Omega \rightarrow E$ a vector measure and $\mu$ : $\Omega \rightarrow \mathbb{K}$ a scalar measure. Then the following statements are equivalent:

(1) $m<<\mu$.

(2) There exists a $g \in \mathcal{L}(\mu, E)$ such that $m=\mu \otimes g$.

Proof. (2.) $\Rightarrow$ (1.) It is directed from Lemma 3.7 and Lemma 3.6.

$(1.) \Rightarrow\left(2\right.$.) By the assumption, for all $a \in X$, there exists $e_{a} \in E$ such that for all $p \in \Gamma, \mathcal{N}_{m-\mu \otimes e_{a}, p}(a)=0$, .

First of all, let us prove that if $\mathcal{N}_{\mu}(a)>0$, then $e_{a}$ is unique. As usual, suppose that there exists another $w_{a} \in E$ such that

$$
\mathcal{N}_{m-\mu \otimes e_{a}, p}(a)=0=\mathcal{N}_{m-\mu \otimes w_{a}, p}(a), \text { for all } p \in \Gamma .
$$

Then, for a given $\varepsilon>0$, there exists $U \in \Omega, a \in U$ such that

$$
\max \left\{\|U\|_{m-\mu \otimes e_{a}, p},\|U\|_{m-\mu \otimes w_{a}, p}\right\} \leq \varepsilon .
$$

Note that, in general, $p(e) \mathcal{N}_{\mu}(a)=\mathcal{N}_{\mu \otimes e, p}(a)$. Thus, if $\mathcal{N}_{\mu}(a)>0$, then

$$
\begin{aligned}
p\left(e_{a}-w_{a}\right) \mathcal{N}_{\mu}(a) & \leq p\left(e_{a}-w_{a}\right)\|U\|_{\mu}=\sup _{V \in \Omega, V \subset U}|\mu(V)| p\left(e_{a}-w_{a}\right) \\
& =\sup _{V \in \Omega, V \subset U} p\left(\mu \otimes e_{a}(V)-\mu \otimes w_{a}(V)\right) \\
& =\sup _{V \in \Omega, V \subset U} p\left(\mu \otimes e_{a}(V)-m(V)+m(V)-\mu \otimes w_{a}(V)\right) \\
& =\sup _{V \in \Omega, V \subset U} \max \left\{p\left(\mu \otimes e_{a}(V)-m(V)\right), p\left(m(V)-\mu \otimes w_{a}(V)\right)\right\} \\
& =\max \left\{\|U\|_{m-\mu \otimes e_{a}},\|U\|_{m-\mu \otimes w_{a}}\right\}<\varepsilon
\end{aligned}
$$


Thus, $p\left(e_{a}-w_{a}\right)=0$. Since $p$ is arbitrary, we conclude $e_{a}=w_{a}$.

Now, let us define the function $g: X \longrightarrow E$, by

$$
g(a)= \begin{cases}e_{a}, & \mathcal{N}_{\mu}(a)>0 \\ 0, & \mathcal{N}_{\mu}(a)=0\end{cases}
$$

By the uniqueness of $e_{a}, g$ is well defined.

The next step is to prove that $g$ is $\mu$-integrable. By Theorem 3.3, it is enough to prove that

- $g$ is continuous on each $X_{t}, t>0$.

- For a given $\delta>0$ and $p \in \Gamma$, there exists a compact subset $Q$ of $X$, contained in some $X_{t}$, such that $p(f) \mathcal{N}_{\mu} \leq \delta$ off $Q$.

Take $t>0, p \in \Gamma$ and $\varepsilon>0$. If $a \in X_{t}$ and $\mathcal{N}_{m-\mu \otimes g(a), p}(a)=0$, by Lemma 3.6,

$$
\underset{U \rightarrow a}{\operatorname{LIM}}[m(U)-\mu \otimes g(a)(U)]=0 .
$$

So, for these $t$ and $\varepsilon$, there exists $U \in \Omega, a \in U$, such that

$$
V \in \Omega, V \subset U \Rightarrow p(m(V)-\mu \otimes g(a)(V)) \leq \frac{\varepsilon t}{2} .
$$

Now, if $b \in U \cap X_{t}$ and since $\mathcal{N}_{m-\mu \otimes g(b), p}(b)=0$, then we may choose $V_{0} \in \Omega$, $V_{0} \subset U, b \in V_{0}$ such that

$$
V \in \Omega, V \subset V_{0} \Rightarrow p(m(V)-\mu \otimes g(b)(V)) \leq \frac{\varepsilon t}{2} .
$$

Thus,

$$
\begin{aligned}
p(g(a)-g(b)) \frac{t}{2} & \leq p(g(a)-g(b)) \mathcal{N}_{\mu}(b) \leq p(g(a)-g(b))\left\|V_{0}\right\|_{\mu} \\
& =p(g(a)-g(b)) \sup _{V \in \Omega, V \subset V_{0}}|\mu(V)| \\
& =\sup _{V \in \Omega, V \subset V_{0}} p(\mu \otimes g(a)(V)-\mu \otimes g(b)(V)) \\
& =\sup _{V \in \Omega, V \subset V_{0}} \max \left\{\begin{array}{l}
p(m(V)-\mu \otimes g(a)(V)) \\
p(m(V)-\mu \otimes g(b)(V))
\end{array}\right\} \leq \frac{\varepsilon t}{2}
\end{aligned}
$$

which implies the continuity of $g$ in $X_{t}$.

In order to prove the second condition for $g$, let $\delta>0, p \in \Gamma$ and define the set $Q=\left\{x \in X: \mathcal{N}_{m, p}(x) \geq \delta\right\}$. We claim that $Q$ is the compact we need. Note that $\mathcal{N}_{m-\mu \otimes g(x), p}(x)=0$ implies $\mathcal{N}_{m, p}(x)=\mathcal{N}_{\mu \otimes g(x), p}(x)$. If $x \notin Q$, then

$$
p(g(x)) \mathcal{N}_{\mu}(x)=\mathcal{N}_{\mu \otimes g(x), p}(x)=\mathcal{N}_{m, p}(x)<\delta \text {. }
$$

Therefore, we only need to prove that $Q$ is contained in some $X_{t}$. Take $a \in Q$; again by Lemma 3.6, there exists $U_{a} \in \Omega, a \in U_{a}$ such that

$$
V \in \Omega, V \subset U_{a} \Rightarrow p(m(V)-\mu \otimes g(a)) \leq \frac{\delta}{2} .
$$

By the compactness and by the fact that

$$
Q \subset \bigcup_{a \in Q} U_{a}
$$


we may choose a finite collection $\left\{U_{a_{1}}, \cdots U_{a_{n}}\right\}$ in $\Omega$ such that

$$
Q \subset \bigcup_{i=1}^{n} U_{a_{i}} \text {. }
$$

Thus, if $V \in \Omega, V \subset U_{a_{i}}$, then

$$
\begin{aligned}
p(m(V)) & =p\left(m(V)-\mu \otimes g\left(a_{i}\right)(V)+\mu \otimes g\left(a_{i}\right)(V)\right) \\
& \leq \max \left\{p\left(m(V)-\mu \otimes g\left(a_{i}\right)(V)\right), p\left(\mu \otimes g\left(a_{i}\right)(V)\right)\right\} \\
& \leq \max \left\{\frac{\delta}{2}, p\left(\mu \otimes g\left(a_{i}\right)(V)\right)\right\} .
\end{aligned}
$$

If we choose $M \geq \max \left\{p\left(g\left(a_{i}\right)\right): 1 \leq i \leq n\right\}$ and $t=\delta M^{-1}$, then $Q \subset X_{t}$. In fact, take $a \in Q$ and $U_{a_{i}}$ such that $a \in U_{a_{i}}$. For an arbitrary $V \in \Omega, V \subset U_{a_{i}}$ we have

$$
\begin{aligned}
p(m(V)) & \leq \max \left\{\frac{\delta}{2}, p\left(\mu \otimes g\left(a_{i}\right)(V)\right)\right\}=\max \left\{\frac{\delta}{2},|\mu(V)| p\left(g\left(a_{i}\right)\right)\right\} \\
& \leq \max \left\{\frac{\delta}{2},|\mu(V)| \delta t^{-1}\right\} .
\end{aligned}
$$

and thus,

$$
\begin{aligned}
\delta & \leq \mathcal{N}_{m, p}(a)=\overline{L I M}_{U \rightarrow a} p(m(U)) \\
& \leq \max \left\{\frac{\delta}{2}, \delta t^{-1} \overline{L I M}_{U \rightarrow a}|\mu(U)|\right\} \\
& =\delta t^{-1} \overline{L I M}_{U \rightarrow a}|\mu(U)|=\delta t^{-1} \mathcal{N}_{\mu}(a)
\end{aligned}
$$

which means

$$
\mathcal{N}_{\mu}(a) \geq t
$$

As a consequence, $g$ is $\mu$-integrable.

Hence, by Lemma 3.7,

$$
\underset{U \rightarrow a}{\operatorname{LIM}}[\mu \otimes g(U)-\mu \otimes g(a)(U)]=0 .
$$

which implies that for every $p \in \Gamma, \mathcal{N}_{\mu \otimes g-\mu \otimes g(a), p}(a)=0$.

Therefore,

$$
\mathcal{N}_{m-\mu \otimes g, p}(a) \leq \max \left\{\mathcal{N}_{m-\mu \otimes g(a), p}(a), \mathcal{N}_{\mu \otimes g-\mu \otimes g(a), p}(a)\right\}=0 .
$$

In other words, $\mathcal{N}_{m-\mu \otimes g, p} \equiv 0$ or, equivalently, $m=\mu \otimes g$.

\section{REFERENCES}

1. J.N. Aguayo and T.E. Gilsdorf, Non-archimedean Vector Measures and Integral Operators, Lecture Notes, Marcel Dekker, Inc., Vol 222, 2001.

2. A.K. Katsaras, Duals Of Non-Archimedean Vector-Valued Functions Spaces, Bull. Greek Math. Soc. 22 (1981), 25-43.

3. A.K. Katsaras, The Strict Topology In Non-Archimedean Vector-Valued Function Spaces, Indag. Math. 46 (1984), 189-201.

4. A.F. Monna and T.A. Springer, Integration Non-archimedienne, Indag. Math. 25 (1963), $634-653$. 
5. M. Moraga and J.N. Aguayo, A Radon-Nikodym theorem in the non-Archimedean setting, Proyecciones 20 (2001), no. 3, 263-279.

6. W.H. Schikhof, A Radon-Nikodym theorem for non-archimedean integrals and absolutely continuous measures on groups, Indag. Math. 33 (1971), 78-85.

7. A.C.M. van Rooij, Non-archimedean Functional Analysis, New York, Marcel Dekker, 1978.

8. A.C.M. van Rooij and W.H. Schikhof, Non-Archimedean integration theory, Indag. Math. 31 (1969), 190-199.

Dpto de Matemática, Fac. de Cs. Fís y Mat., Universidad de Concepción, CHILE.

E-mail address: jaguayo@udec.cl

E-mail address: camiloperez@udec.cl 